\title{
Study of an array of two circular jets impinging on a flat surface
}

\author{
Ștefan-Mugur Simionescu ${ }^{1, *}$, Nilesh Dhondoo ${ }^{1}$ and Corneliu Bălan ${ }^{1}$ \\ ${ }^{1}$ REOROMGroup, Department of Hydraulics, Hydraulic Machinery and Environmental Engineering, Faculty of Power Engineering, \\ University Politehnica of Bucharest, Romania
}

\begin{abstract}
In this study, the flow characteristics of an array of two circular, laminar air jets impinging on a smooth solid wall are experimentally and numerically investigated. Direct visualizations using high speed/resolution camera are performed. The evolution of the vortical structures in the area where the jet is deflected from axial to radial direction is emphasized, as well as the interaction between the two jets. A set of CFD numerical simulations in 2D flow domains are performed by using the commercial software Fluent, in the context of Reynolds-averaged Navier-Stokes (RANS) modeling. The numerical resultsare compared and validated with the experiments. The vorticity number is computed and plotted at two different positions from the jet nozzle, and a study of its distribution gives a clue on how the jets are interacting with each other in the proximity of the solid wall.
\end{abstract}

\section{Introduction}

Due to their practical importance, the study of impinging jets has received considerable attention in the last years. Of particular interest are the changes brought to the jet dynamics when modifying some important flow characteristics, as the position of the jet nozzle with respect to the impingement wall, shape of the nozzle, number of jet nozzles, wall roughness and structure.

The behaviour of impinging jets in the vicinity of solid walls has been extensively investigated experimentally. A survey of literature on flow characteristics of a single air jet impinging on a flat plate was made in 1970 by Gauntner, Livingood\&Hrycak[1], focused mainly on basic flow statistics. Tahsini and Mousavi[2] provide a classification of different parameters that characterize impinging slot jets. The effects of the jet Reynolds number, jet confinement, nozzle-to-plate distance and turbulent intensity are discussed.

Later experimental studies brought up the phenomenon of heat transfer between a solid surface and a fluid jet, as the work of Jambunathan et al. [3] on single circular jet impingement.Hadziabdic and Hanjalic[4]showed results on turbulent heat transfer from an impinging slot/round jet. Geers et al. [5], [6], studied turbulent structures and heat transfer from arrays of impinging jets.

Despite significant progress in understanding macro- and microscopic phenomena occurring in impinging jets, many problems remain unsolved because of limitations in experimental measuring techniques. A solution to this problem appeared more than three decades ago, when numerical simulations of fluid flows became possible along with the advance on the market of powerful computing systems. Computational Fluid Dynamics (CFD), with its potential to solve the dynamics of a flow in both space and time, is nowadays used as a valuable instrument that provides information and even complements the experimental results.

The authors of the present study conducted a set of CFD simulations on the heat transfer between laminar impinging air jets and solid surfaces [7]. For turbulent flows, many numerical results can be found in the literature for this field of study, involving Reynoldsaveraged Navier-Stokes (RANS) or Large Eddy Simulations (LES). Some significant examples are the studies by Jaramillo et al. [8] comparing the results given by different turbulence models, or Kalitzin et al. [9] studying the near-wall behaviour of RANS turbulence models.Other authors have developed new computational turbulence models, as in the case of Kubacki and Dick [10], [11] who used a k- $\omega$ based hybrid RANS/LES model for simulating plane impinging jets.

More recently Direct Numerical Simulations (DNS) of complex flows have become possible, solving the whole range of spatial and temporal scales of turbulence. Friedrich et al. [12] studied incompressible turbulent flows by using the same technique.

The aim of the present work is to study the time evolution of an array of two jets formed by circular orifices, impinging on a solid smooth wall, from both experimental and numerical point of view. The experiments provide a validation mean for the CFD results, which are then extended to extract new data. Detailed distributions of significant flow parameters in the jet impact region are inferred from the numerical tests.

\footnotetext{
*Corresponding author: stefan_simionescu@yahoo.com
} 


\section{Theoretical aspects}

For the case of a single unconfined jet impinging on a flat surface, most authors mention three main regions: free jet region, stagnation region and wall jet region [1], [3], [13]. For the array of two circular jets, each jet first develops as a free jet after emerging, then enters its stagnation region, close to the solid wall. After hitting the wall, each jet developsas a wall jet in all the radial directions where it is not influenced by the neighboring jet. Between the two jets, a constant mixing zone can be found, in form of two counter-rotating vortices. A view of two circular jets impinging on a solid wall is presented in Fig. 1, taken in the moments after the circular jets hit the wall.

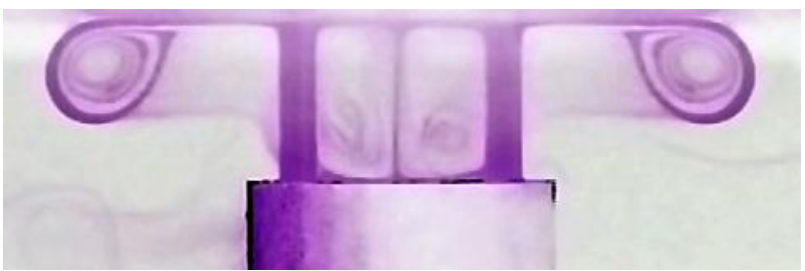

Fig. 1. Visualization of an array of two circular jets impinging on a flat surface.

The flow characteristics of an array of two impinging jets depend upon a number of parameters: diameter of the two jets $d_{1}$ and $d_{2}$, Reynolds number of each jet $R e$, nozzle-to-wall distance $z$, distance between the jet nozzles, structure and roughness of the impingement surface, jet configuration and orifice shape and profile. The Reynolds number $R e$ of a circular jet is defined as $R e=\rho v d / \eta$, with $\rho$ the fluid density, $v$ mean velocity of the jet and $\eta$ the fluid viscosity.

The flow regime presents a fundamental importance for the study of impinging jets. Comprehensive studies were written on the subject, but some still show contradictory results. A classification of circular gaseous jets, based on the Reynolds number $R e$ is stated by Gardon and Akfirat[14], and separates laminar from turbulent jets at $R e \cong 1000$. This study distinguishes laminar jets for $R e<1000$, transition jets or semi-turbulent jets for $1000<R e<3000$ and fully turbulent jets for $R e>$ 3000 .

The concept of vorticity is related to vortices and vortical flows[15], [16]. The nondimensionalvorticity numberWo is computed as the ratio between vorticity magnitude $\boldsymbol{\Omega}$ and strain rateD[16]. Whithin the present study it will be usedto quantify the existence vortices and vortical structures within the fluid domain.

\section{Experimental set-up}

As first step of the study, a set of direct visualizations was performed. The experimental facility used for the present study is sketched in Fig. 2. The two circular jet nozzles have a fixed $4.8 \mathrm{~mm}$ diameter, and are set at a distance equal to 4 times the jet diameter from each other. The impingement surface used for the tests is a

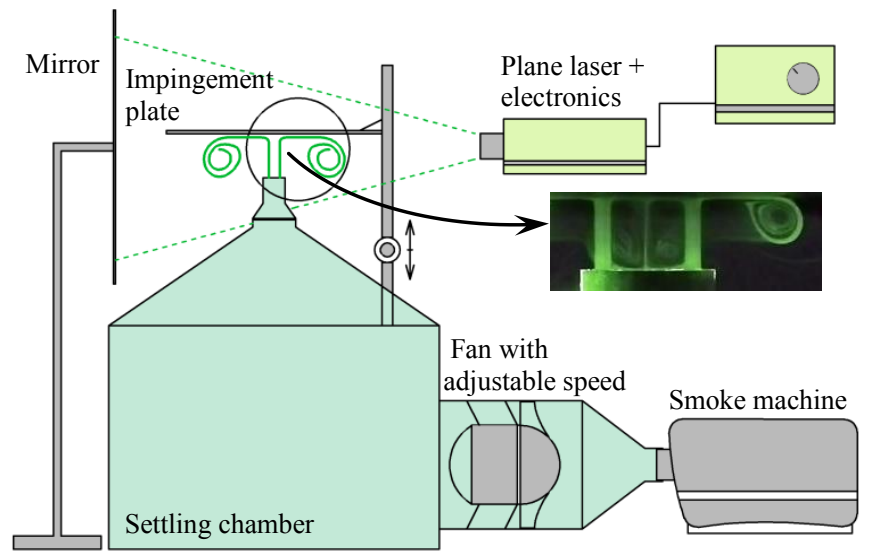

Fig. 2. Schematic view of the experimental set-up, with detail of the impingement zone.

smooth plexiglasplate. The nozzle-to-plate distance $h / d$ is fixed for all the experiments, with a value of 4 . The Reynolds number used for all the experimental and numerical tests is $R e=200$. The settling chamber has a volume of $170 \mathrm{dm}^{3}$. The air is seeded with particles by use of a smoke machine Showtec Atmos 1000. The seeded air is sent inside the chamber and then through the jet nozzle by a fan with adjustable speed. A continuous laser with $532 \mathrm{~nm}$ wavelength, equipped with a cylindrical lens to convert the beam in a planar sheet, is then lighting the impinging jet through its axis in order to make it visible. A mirror reflects back the laser beam to increase the lighting power in the area where the jets hit the wall. The dynamics and interaction of the impinging jets is recorded with a Nikon $1 \mathrm{~J} 5$ camera at $400 \mathrm{fps}$ and a resolution of $800 \times 296$ pixels. The camera is placed perpendicular to the plane lighted by the laser sheet, so that it focuses the plane that passes through the center of the two nozzles.

The mean jet velocity is measured experimentally as the distance travelled by the fluid in a certain time period. The distance between the jet nozzle and the solid wall isrigorously set and is taken as reference, as for the time, it is obtained from the camera framerate.

\section{Numerical study}

In parallel with the experimental tests, a set of numerical simulations was performed, in order to validate and further analyze a case similar to the one experimentally tested. The numerical simulations were performed by using the commercial software ANSYS Fluent, in the context of Reynolds-averaged Navier-Stokes (RANS) modeling. As the Reynolds number used for all the simulations is low $(R e=200)$ the laminar model was used. The Fluent solver is based on a finite-volume solution of the mean momentum, energy and turbulent transport equations. The scheme selected to perform the computations was second order upwind.

The numerical cases were modeled in a two-dimensional flow domain, built and discretized by using the software program Gambit. 


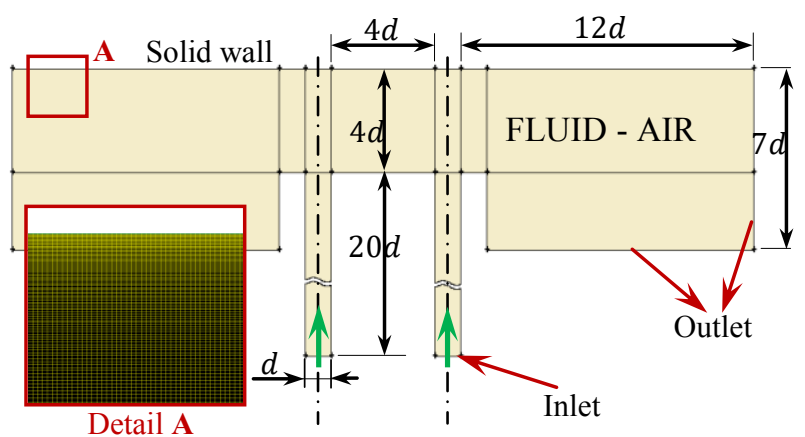

Fig. 3. Numerical flow domain and boundary conditions, with detail of the mesh.

A schematic view of the computational domain with boundary conditions is presented in Fig. 3. The outlet boundary is placed at a sufficiently large radial distance, so that the error from applying the outlet-pressure condition will not affect the region of interest. According to Dairay et al. [17], the radial expansion of the flow domain was chosen at $12 d$. The mesh has 198000 cells, and was built with special attention in the region close to the impingement plate, where a further refinement was done.

\section{Results and discussion}

As the two jets emerge and approach the solid wall, its presence begins to affect the velocity field. A stagnation region appears close to the impingement point of each jet. Due to the presence of the impermeable wall, the axial velocity drops fast, at the same time with an increase of the static pressure [1]. After hitting the wall, the jets take radial direction and a wall-jet is formed downstream.

A time evolution of the two circular jets impinging on the flat surface is presented in Fig. 4. The turbulence induced between the two jets is highlighted by the two counter-rotating vortices found in this space. Perturbations present in the flow, as wall roughness, pressure pulsations etc., make these vortices change shape and position in time and dissipate a part of the energy from the jets.

The two jets start to influence each other from the first moments after emerging from the nozzles.So, they tend to move away from each other.This aspect is more visible in the numerical simulations, due to the limitations brought by the use of a two-dimensional flow domain instead of a physical three-dimensional one.

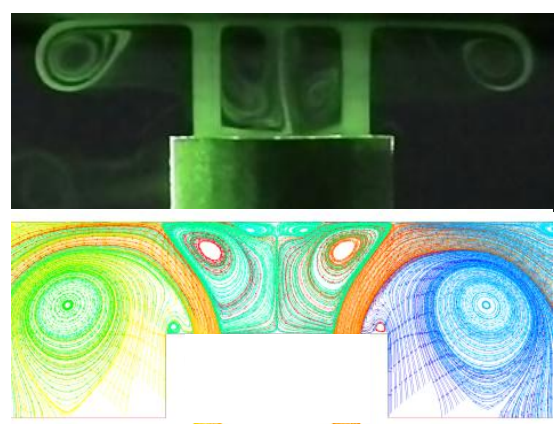

Fig. 5. Comparison between experimental and numerical results, at $400 \mathrm{~ms}$ after the jets emerge.

A direct flow visualization taken at $400 \mathrm{~ms}$ after the jets emerge from the nozzlesis compared with the corresponding numerical result in term of velocity pathlines - Fig. 5. A good agreement is found in terms of vortex core radial development, although for the numerical simulations the general shape of the jets is altered by the influence exerted by the two jets to each other. As the flow Reynolds number is low, the direct experimental visualizations show the two jets hitting the flat surface almost perpendicular. On the contrary, in the numerical resultthe jets tend to tilt and diverge as they approach the solid wall.

In Fig. 6a, the contours of vorticity number are shown at the time moment of $400 \mathrm{~ms}$ after jet emerge. In Fig. 6b, a vorticity number graph is plotted on the two lines shown: first continuous red line at a distance of $2 d$ from the jet nozzle, and second dark blue line at a distance of $3 d$ from the nozzle. This correlation between vorticity number contours in the domain and value on the two lines gives a clue on how the jets are interacting with each other,in the presence of the solid wall.

\section{Conclusion}

A set of flow visualizations on the behavior of an array of two circular, laminar air jets impinging on a smooth flat surface were conducted to show the non-stationary jet dynamics of the flow. The analysis of the three main zones crossed by the fluid (free jet, stagnation region and radial wall jet) show a specific dynamics for the case of flow emerging from two jet nozzles.

The stagnation region is the one with the highest velocity gradients, as the jet is constrained by the wall to take radial direction. Downstream of this region, the flow takes two different behaviors: forms a vortex ring that
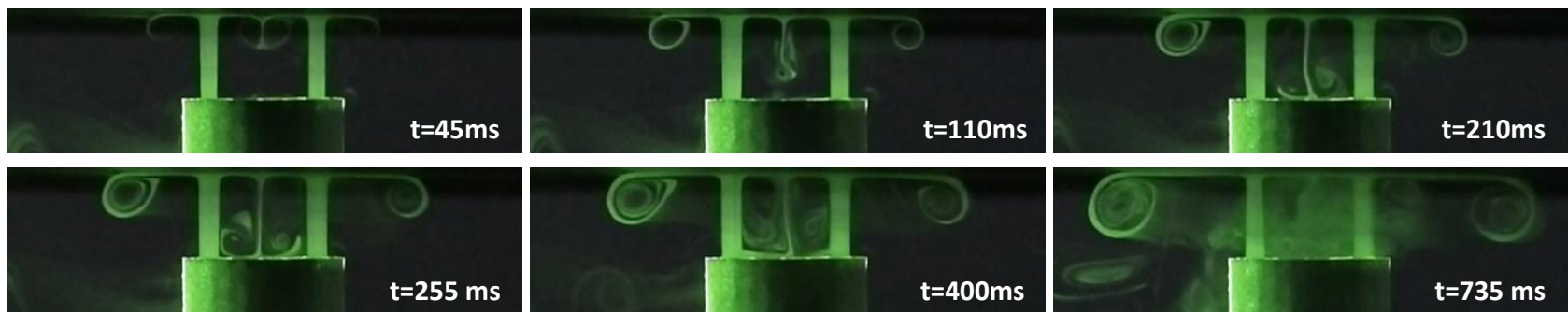

Fig. 4. Time evolution ofthe two circular jets impinging on flat surface:time measured from emergence of jets. 
a)

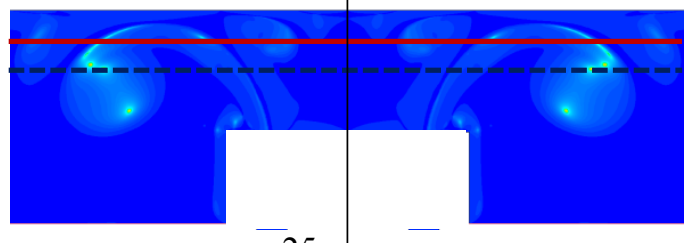

b)

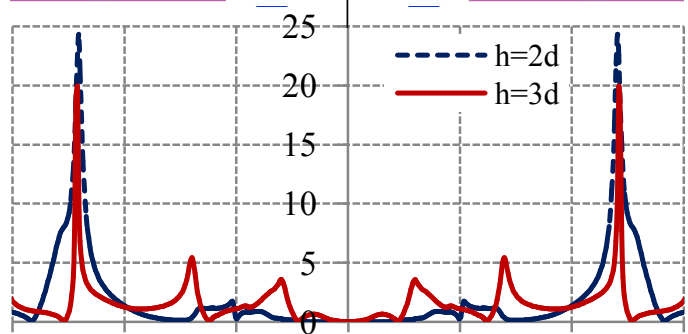

$\begin{array}{lll}-0.048 & -0.032 & -0.016\end{array}$

0

$0.016 \quad 0.032$

0.048

Fig. 6.Contours of vorticity number at $400 \mathrm{~ms}$ after jets emerge (a) and vorticity number on two lines at $h=2 d$ and $h=3 d$ from nozzle exit (b).

evolvesradially on the outer side of the two nozzles, as between the nozzles two counter-rotating vortices rollconstantly and dissipate a part of the jet energy.

In parallel with the experiments, a CFD study in the same flow conditions was performed in ANSYS Fluent. The evolution of the vortex rings observed in the numerical simulations, emerging after the jets hit the impermeable wall, agrees in terms of vortex radial expansion with the one found experimentally. After the validation is performed, a distribution of the vorticity number is plotted on two different lines.

After performing this work, the opportunity arises for new numerical simulations on the topic, using threedimensional flow domains for a better reproduction of the physical processes. Also, a set of tests involving turbulent impinging jets can bring the opportunity for a further observation of the turbulence phenomena.

This work was partially supported by a grant of the Romanian National Authority for Scientific Research, CNCS, UEFISCDI, PHANTOM - Passive flow control for heat and mass transfer enhancement of impinging jets PN-III-P4-ID-PCE-2016-0758, and by a grant of the Romanian space agency ROSA, QUEST Advanced air diffusion system of the crew quarters for the ISS and deep space habitation systems, STAR-CDI-C3-2016-577.

\section{References}

1. J. W. Gauntner, J. N. B. Livingood, and P. Hrycak, NASA Technical Note, Washington, DC (1970).

2. A. M. Tahsini and S. T. Mousavi, World Acad. Sci. Eng. Technol.6, 1201-1205 (2012).

3. K. Jambunathan, E. Lai, M. A. Moss, and B. L. Button, Int. J. Heat Fluid Flow13, 106-115 (1992).

4. M. Hadžiabdić and K. Hanjalić, J. Fluid Mech.596, 221-260 (2008).

5. L. F. G. Geers, M. J. Tummers, and K. Hanjalić, Exp. Fluids36, 946-958 (2004).

6. L. F. G. Geers, K. Hanjalic, and M. J. Tummers, J. Fluid Mech.546, 255-284 (2006).

7. Ş.-M. Simionescu and C. Bălan, Energy Procedia85, 481-488 (2016).

8. J. E. Jaramillo, K. Claramunt, J. Cadafalch, and C. D. . Pérez-Segarra, Eur. Congr. Comput. Methods Appl. Sci. Eng. ECCOMAS, 1-20 (2004).

9. G. Kalitzin, G. Medic, G. Iaccarino, and P. Durbin, J. Comput. Phys. 204, 265-291 (2005).

10. S. Kubacki and E. Dick, Int. J. Heat Fluid Flow31, 862-878 (2010).

11. S. Kubacki, J. Rokicki, and E. Dick, Int. J. Heat Fluid Flow44, 596-609 (2013).

12. R. Friedrich, T. J. Hüttl, M. Manhart, and C. Wagner, Comput. Fluids30, 555-579 (2001).

13. N. Rajaratnam and K. A. Mazurek, J. Hydraul. Res.43, 689-695 (2005).

14. R. Gardon and J. C. Akfirat, Int. J. Heat Mass Transf.8, 1261-1272 (1965).

15. P. Brancher, J. M. Chomaz, and P. Huerre, Phys. Fluids6, 1768-1774 (1994).

16. H. J. Lugt, Introduction to vortex theory. Vortex Flow Press (1996).

17. T. Dairay, V. Fortuné, E. Lamballais, and L. E. Brizzi, Int. J. Heat Fluid Flow764, 362-394 (2015). 